\title{
INCORRECTLY POSED BVPS FOR ELASTIC HALF- PLANE IN TERMS OF STRESS AND DISPLACEMENT ORIENTATIONS
}

\author{
A.N. GALYBIN ${ }^{1}$ \\ ${ }^{1}$ The Schmidt Institute of Physics of the Earth (IPE RAS), Moscow, Russia.
}

\begin{abstract}
This article presents the general approach to incorrectly posed boundary value problems of the plane theory of elasticity with the boundary conditions formulated through the directions of displacement vectors and principal directions of the stress tensor. The approach is aimed at studying the solvability of problems of this type and allows one to determine the maximum number of arbitrary parameters on which the solution of the problem depends. We report analytical solutions for three particular formulations for the case of elastic half-plane and propose a numerical approach for solving the problems of this type for arbitrary contours.

Keywords: boundary value problems, complex potentials, plane elasticity, singular integral equations.
\end{abstract}

\section{INTRODUCTION}

This study is an extended version of the papers [1], [2] that have been published recently in regard to incorrectly posed problems of plane elasticity that use the boundary conditions (BC) formulated in terms of stress and displacement orientations. The first paper [3] on this topic has been published in 1999 and assumed the BC posed on the principal stress directions and the curvature of the stress trajectories on the contour (expressed through the tangential and normal derivative of the principal directions). The approach to these problems involved reduction to a system of singular integral equations followed by reduction of the latter to the Riemann boundary value problem. The main results of [3] are briefly discussed in this paper in order to detail the differences in the corresponding integral equations obtained for formulations discussed in [1]-[3].

The motivation of these studies originated from geomechanics where the boundary values of stresses and/or displacements are often unknown on the boundary, which makes it impossible to apply classical formulations accepted in solid mechanics. However even incomplete information, such as orientations of principal stresses or displacements can serve as possible boundary conditions. Accordingly, several non-classical formulations of elastic boundary value problems, BVP, employing BC in terms of orientations of principal stresses, displacements or tractions have been investigated in [1]-[8]. The mentioned studies are mostly dedicated to the investigation of solvability of the BVP with incomplete BC, which leads to non-unique solutions. The latter is proved by using the following approach. The complex potentials are presented by the Cauchy integrals with unknown densities. Their boundary values are found by employing the Sokhotski-Plemelj formulas. These are used to express the orientations of tractions, principal stresses and displacements on the contour in the form that contains two unknown complex valued functions. Further on, one of these functions is excluded by imposing the condition of continuity of the stress vector across the contour. Then by using two scalar BC in terms of the orientations one can derive a system of two real singular equations, SIE. These are combined into one linear complex SIE of the general form

1 ORCID: http://orcid.org/0000-0003-3948-5685 
that contains the unknown function, its conjugate, the singular and regular integrals and their conjugates. An approach suggested by Gakhov [9] is used for analysing the solvability of the complex SIE. It assumes separation of the dominant part of the complex SIE followed by its conversion into the equivalent Riemann problem. The solvability of the latter depends on the index of the coefficient of the Riemann problem that is calculated through the increment of its argument (divided by $2 \pi$ ) in traversing the contour anticlockwise. If the index is positive then the Riemann problem has a finite number of solutions that is proportional to the index, otherwise the BVP does not have solutions in the class of holomorphic functions.

It is shown in [1]-[8] that the dominant SIE corresponds to the case of elastic half-plane regardless of the $\mathrm{BC}$ used. Therefore, we further consider the dominant SIE only. However, in contrast to the previously used approach we deal with two real SIEs. The reason behind this is to build the successive solutions of the equations in the system for the problems that do not directly allow for the application of the technique mentioned above because of the presence of the derivatives of the unknown functions in the complex dominant SIE.

\section{PRELIMINARIES}

The general solution of plane elastic problems for isotopic homogeneous media is expressed in terms of the Kolosov-Muskhelishvili formulas [10] by two holomorphic functions (complex potentials) $\Phi(\mathrm{z})$ and $\Psi(\mathrm{z})$ of a complex variable $z=x_{1}+i x_{2}$ and have the form (no body forces)

$$
\begin{aligned}
& P \equiv \frac{\sigma_{22}+\sigma_{11}}{2}=\Phi(z)+\overline{\Phi(z)}, \quad D \equiv \frac{\sigma_{22}-\sigma_{11}}{2}+i \sigma_{12}=\bar{z} \Phi^{\prime}(z)+\Psi(z) \\
& 2 G W \equiv 2 G\left(u_{1}+i u_{2}\right)=\kappa \phi(z)-z \overline{\Phi(z)}-\overline{\psi(z)}, \quad \Phi(z)=\phi^{\prime}(z), \quad \Psi(z)=\psi^{\prime}(z) .
\end{aligned}
$$

Here $P$ is the mean stress (a harmonic function), $D$ is the complex stress deviator (a bi-holomorphic function), $\sigma_{i j}$ are the components of the plane stress tensor $(i, j=1,2), W$ is the complex displacement vector, $u_{i}(i=1,2)$ are the displacement components, $G$ is the shear modulus, $\kappa=3-4 v$ for the plain stress and $\kappa=(3-v) /(1+v)$ for the plane stress conditions, $v$ is Poisson's ratio. Hereafter, we do not show the arguments of the stresses/displacements, but keep them for the potentials where necessary to distinguish between boundary and internal points.

The complex potential $\varphi(\mathrm{z})$ and its derivatives can be expressed in the form of the Cauchytype integrals as follows:

$$
\phi(z)=\frac{1}{2 \pi i} \int_{\Gamma} \frac{g(t)}{t-z} d t, \quad \Phi(z)=\frac{1}{2 \pi i} \int_{\Gamma} \frac{g^{\prime}(t)}{t-z} d t, \quad \Phi^{\prime}(z)=\frac{1}{2 \pi i} \int_{\Gamma} \frac{g^{\prime \prime}(t)}{t-z} d t
$$

where $\Gamma$ is a smooth contour (closed or open) in the complex plane and $g(t)$ is a complexvalued function referred to as the density of the complex potential. For $\psi(z)$ and $\Psi(z)$ it is convenient to use the following presentation:

$$
\psi(z)=\frac{1}{2 \pi i} \int_{\Gamma} \frac{h(t)-\bar{t} g^{\prime}(t)}{t-z} d t, \quad \Psi(z)=\frac{1}{2 \pi i} \int_{\Gamma} \frac{h^{\prime}(t)-\bar{t} g^{\prime \prime}(t)-e^{-2 i \vartheta(t)} g^{\prime}(t)}{t-z} d t
$$

where $h=h(t)$ is another complex-valued function $e^{i \vartheta(\zeta)}=\frac{d \zeta}{d s}=\zeta^{\prime}, \quad \zeta \in \Gamma, \vartheta$ is the angle between the tangent and the $x_{1}$-axis, $s$ is the arc length. 
The boundary values of the complex potentials are found by the Sokhotski-Plemelj formulas [9]

$$
2 \phi^{ \pm}= \pm g+\mathbf{S}(g), \quad 2 \psi^{ \pm}= \pm h \mp \bar{\zeta} g^{\prime}+\mathbf{S}\left(h-\bar{t} g^{\prime}\right)
$$

where the superscripts designate the boundary values for the interior $(+)$ and exterior $(-)$ domains with respect to the contour $\Gamma$ and the following notation for the singular integral is introduced

$$
\mathbf{S}(g)=\frac{1}{\pi i} \int_{\Gamma} \frac{g(t)}{t-\zeta} d t, \quad \zeta \in \Gamma, \quad \mathbf{S}[\mathbf{S}(g)]=g .
$$

By using (4) one obtains the boundary values of the stress and displacement functions

$$
\begin{gathered}
2 P^{ \pm}= \pm\left(g^{\prime}+\bar{g}^{\prime}\right)+\mathbf{S}\left(g^{\prime}-\bar{g}^{\prime}\right)+\mathbf{R}_{2}\left(\bar{g}^{\prime}\right), \\
2 D^{ \pm}= \pm\left(h^{\prime}-e^{-2 i \vartheta} g^{\prime}\right)+\mathbf{S}\left(h^{\prime}-e^{-2 i \vartheta} g^{\prime}\right)+\mathbf{R}_{1}\left(g^{\prime}\right) \\
2 G W^{ \pm}= \pm(\kappa g-\bar{h})+\mathbf{S}(\kappa g+\bar{h})-\overline{\mathbf{R}_{1}(g)}-\mathbf{R}_{2}(\bar{h})
\end{gathered}
$$

where two regular integral operators are introduced as follows:

$$
\mathbf{R}_{1}(g)=-\mathbf{S}\left((\bar{t}-\bar{\zeta}) g^{\prime}\right), \quad \mathbf{R}_{2}(\bar{g})=\overline{\mathbf{S}(g)}+\mathbf{S}(\bar{g}) .
$$

It should be emphasized that the unknown functions $g$ and $h$ are not independent of each other and a certain relationship between them should be imposed depending on BC of the problem considered. If one selects $h=\kappa \bar{g}$ then non-integral terms in (8) disappear, which means continuity of the displacement vector across the contour. If $h=-\bar{g}$ (respectively, $\left.h^{\prime}=-e^{-2 i \vartheta} \bar{g}^{\prime}\right)$ then the stress vector is continuous across the contour and the expression $\sigma=P+e^{-2 i \vartheta} \bar{D}$ derived from (6) and (7) does not contain non-integral terms and has the form

$$
2 \sigma^{ \pm}=2 \sigma=2 \mathbf{S}\left(g^{\prime}\right)-e^{-2 i \vartheta} \overline{\mathbf{S}\left(\left(e^{-2 i \vartheta(t)}-e^{-2 i \vartheta(\zeta)}\right)\left(g^{\prime}+\bar{g}^{\prime}\right)\right)}+e^{-2 i \vartheta} \overline{\mathbf{R}_{1}\left(g^{\prime}\right)}-\mathbf{R}_{2}\left(g^{\prime}\right)
$$

where the second term in the right-hand side is not singular. It is evident that if the stresses on the contour are given then (10) represents a complex SIE for the determination of the unknown complex-valued function $g^{\prime}$ (as well as (8) can be considered as a SIE for the second fundamental problem). It can be shown that by simple manipulations (8) and (10) can be reduced to those forms reported in Linkov [11].

One can use (6)-(8) in order to derive SIEs for incorrectly posed BVPs considered in the previous studies [1]-[8]. It is worth noting that as shown by Gakhov [9], the regular integral operators do not affect solvability of the complex SIE (excluding some especially designed cases). Therefore, they can be omitted when studying the solvability of a particular boundary value problem for an arbitrary contour. On the other hand, all regular operators in (6)-(10) vanish in the case of half-plane (or become constants in the case of a circle). Moreover, the problems for half-plane/circle can be solved in closed form, which can also be used for the development of numerical methods for arbitrary domains, which is necessary as for certain non-classical formulations the uniqueness is not fulfilled and hence the direct application of 
the standard techniques for solving SIE is not possible. We further consider some typical non-classical BVPs for an isotropic elastic half-plane that lead to different types of SIE and then investigate solvability of these problems.

\section{BOUNDARY VALUE PROBLEMS FOR HALF-PLANE}

\subsection{Formulation}

Let us represent the stress deviator and displacement vector in the form

$$
\begin{gathered}
D=\tau_{\max } \exp (i \alpha), \quad \alpha=\arg D=\pi-2 \theta, \quad \tau_{\max }=|D|, \\
W=\omega \exp (i \beta), \quad \omega=|W|, \quad \beta=\arg W .
\end{gathered}
$$

Here $\tau_{\max }=\left(\sigma_{1}-\sigma_{2}\right) / 2 \geq 0$ is the maximum shear stress, $\sigma_{1}, \sigma_{2}$ are major and minor principal stresses respectively, the angle $\theta=\frac{1}{2} \operatorname{arctg} \frac{2 \sigma_{12}}{\sigma_{11}-\sigma_{22}}$ specifies the principal direction (the angle between $\sigma_{1}$ and the real axis), thus $D=-\tau_{\max } \exp (-2 i \theta) ; W$ is the complex displacement vector, $\omega \geq 0$ and $\beta$ are, respectively, the modulus and the argument of the function $W$.

We consider non-classical formulations in terms of the orientations of stresses and displacements, i.e. assume that the angles $\alpha, \beta$ and their normal derivatives $\alpha_{\mathbf{n}}^{\prime}, \beta_{\mathbf{n}}^{\prime}$ are known on the real axis.

$$
\begin{aligned}
& \left.\arg D^{ \pm}(z, \bar{z})\right|_{z=x+i 0 \pm}=\alpha^{ \pm}(x), \quad|x|<\infty, \\
& \left.\arg W^{ \pm}(z, \bar{z})\right|_{z=x+i 0 \pm}=\beta^{ \pm}(x), \quad|x|<\infty, \\
& \left.\frac{\partial}{\partial y} \arg D^{ \pm}(z, \bar{z})\right|_{z=x+i 0 \pm}=\alpha_{\mathbf{n}}^{\prime \pm}(x), \quad|x|<\infty, \\
& \left.\frac{\partial}{\partial y} \arg W^{ \pm}(z, \bar{z})\right|_{z=x+i 0 \pm}=\beta_{\mathbf{n}}^{\prime \pm}(x), \quad|x|<\infty
\end{aligned}
$$

These four conditions can be combined into three pairs of $\mathrm{BC}$ that will be discussed for the case of an upper half-plane

- $\quad$ BVP1. Problem $\left(\alpha, \alpha_{\mathbf{n}}^{\prime}\right)$ assumes BC (13) and (15).

- $\quad$ BVP2. Problem $\left(\beta, \beta_{\mathbf{n}}^{\prime}\right)$ assumes BC (14) and (16).

- $\quad$ BVP3. Problem $(\alpha, \beta)$ assumes BC (13)-(14).

Note that the indexes \pm above can be dropped when considering a problem for a single half-plane. We will also impose relationships between the unknown functions $g$ and $h$ by assuming either continuity of $\sigma$ or $W$ across the contour, i.e. assuming

$$
h=-\bar{g} \quad \text { or } h=\kappa \bar{g} \text {. }
$$

depending on the BC. The use of either condition in (17) does not narrow the analysis, they provide clear mechanical meaning for analytical continuation of the results onto the entire complex plane through the contour. 
It is worth to notice that the above-formulated problems can be initially reduced to a system of two real SIE (as the conditions (13)-(16) are real) and then combined into a single complex SIE. However even in the latter case the investigation of solvability of the SIE cannot directly use the approach proposed by Gakhov [9] for a complex SIE containing all the terms $g, \bar{g}, \mathbf{S}(g), \overline{\mathbf{S}(g)}$ because the derivatives of the unknown functions are also enter in the formulations of BVP1-BVP3. This requires modification of the Gakhov's method that will be done as for system of two real SIEs as for the complex SIE.

\subsection{Transformation of boundary values}

On the real axis $\Gamma=\{x:|x|<\infty\}$ the singular integral specified in (5) takes the following form:

$$
\mathbf{S}(g)=\frac{1}{\pi i} \int_{-\infty}^{\infty} \frac{g(t)}{t-x} d t, \quad \operatorname{Im} x=0
$$

It satisfies the property

$$
\overline{\mathbf{S}(g)}=-\mathbf{S}(\bar{g})
$$

The boundary values of the functions $D$ and $W$ are found from (7) to (8) by taking into account that $\mathbf{R}_{1}=\mathbf{R}_{2}=0$ and $\vartheta=0$

$$
\begin{gathered}
2 D^{ \pm}= \pm\left(h^{\prime}-g^{\prime}\right)+\mathbf{S}\left(h^{\prime}-g^{\prime}\right) \\
2 G W^{ \pm}= \pm(\kappa g-\bar{h})+\mathbf{S}(\kappa g+\bar{h})
\end{gathered}
$$

Hereafter the superscripts \pm stay for the boundary values for the upper/lower half-planes, respectively, elastic moduli $G$ and $\kappa$ in general can be different for upper/lower half-planes, but we do not address this case and therefore do not use the superscripts \pm for them.

The boundary values of the normal derivatives of the arguments of the functions $D$ and $W$ can be replaced by the boundary values of the derivatives with respect to the complex variable or by its conjugate as explained in [3] for BVP1 and discussed in detail for BVP2.

\section{SOLUTIONS OF THE BOUNDARY VALUE PROBLEMS}

\subsection{BVP1 and BVP2}

BVP1 has been considered in detail by Galybin and Mukhamediev [3] and presented here for the sake of completeness. Investigation of solvability of BVP2 is found in Galybin [1]. Here, we present these solutions together to illustrate the diversity of the approaches. Despite these problems look very similar there exist one essential difference between them. BVP1 is formulated for the bi-holomorphic function $D$, i.e. such a function second derivative of which with respect to the conjugated complex variable vanishes (as evident from the second Kolosov's formula in (1)). The complex-valued function $W$ is not bi-holomorphic but satisfies the second order equation below

$$
\frac{\partial^{2} W}{\partial z^{2}}+\kappa \frac{\partial^{2} \bar{W}}{\partial \bar{z} \partial z}=0
$$


Therefore the results regarding solvability of BVP1 cannot be directly applied to BVP2.

We will briefly present the results obtained for BVP1 and then provide more detailed analysis for BVP2 for the case of an arbitrary contour $\Gamma$ and analytical solutions for the case of half-plane. It is believed that consideration of the general domain will be useful for understanding of the numerical approach that discussed further in Section 5.

\subsubsection{Main results for BVP1}

Let us recall that the system of two real-valued SIE obtained in [3] for BVP1 has the form

$$
\left\{\begin{array}{l}
e^{-i \eta}[\mu+\mathbf{S}(\mu)]-e^{i \eta}[\mu-\mathbf{S}(\mu)]=0, \\
e^{-i \eta}[\lambda+\mathbf{S}(\lambda)]-e^{i \eta}[\bar{\lambda}-\mathbf{S}(\bar{\lambda})]+2 i \alpha_{\mathbf{n}}^{\prime} e^{-i \eta}[\mu+\mathbf{S}(\mu)]=0,
\end{array}\right.
$$

where $\eta=2 \vartheta+\alpha$ is a known angle and $\lambda$ is an auxiliary unknown function expressed through unknown densities $\mu=\operatorname{Re}\left(g^{\prime}\right), \chi=\operatorname{Im}\left(g^{\prime}\right)$ by a certain relationship. It is evident from (23) that the solution of the system can be obtained in two steps. The first SIE is reduced to the homogeneous Riemann problem $A^{+}=-\mathrm{e}^{2 i \eta} A^{-}$, for finding holomorphic function $A$ and $\mu=A^{+}-A^{-}$, followed by the solving the second SIE by reducing it to the non-homogeneous Riemann problem that have the same index $\mathrm{K}_{1}$ as the first one. It is determined as follows:

$$
\mathrm{K}_{1}=\operatorname{Index}(G)=\frac{1}{\pi} \int_{-\infty}^{\infty}\left(2 \vartheta^{\prime}(t)+\alpha^{\prime}(t)\right) d t
$$

The solution of the Riemann problem for $\mathrm{K}_{1}>0$ is found in [9] and, in general, contains a polynomial of the $K_{1}$ degree with complex coefficients. Therefore, the solution of two SIE includes $2 \mathrm{~K}_{1}$ complex coefficients. As the result it has been shown that the system of SIE in (23) depends only on $\mathrm{K}_{1}$ determined by (24) and has $2 \mathrm{~K}_{1}$ arbitrary complex constants (or $4 \mathrm{~K}_{1}$ real), which are not independent due to real valuedness of the function $\mu$ and $\chi$. Thus only half of them are considered as the independent ones as it has been proved in [3]. We do not present the exact formulas for the solution here but have to admit that they are of the form similar to the solution of BVP3 presented further on.

\subsubsection{Transformation of the boundary conditions for BVP2 for an arbitrary contour}

Let us assume that on a smooth contour $\Gamma$ (closed or opened) the orientation of the displacement vector angle $\beta=\beta(\zeta)$ is known together with its normal derivative $\beta_{\boldsymbol{n}}^{\prime}(\zeta)$. The first condition means that $\arg W(\zeta)=\beta(\zeta)$ and leads to the boundary condition of the form:

$$
\operatorname{Im}\left(e^{-\mathrm{i} \beta} W\right)=0 \quad \Leftrightarrow \quad e^{-\mathrm{i} \beta} W=e^{\mathrm{i} \beta} \bar{W}, \quad \zeta \in \Gamma .
$$

This equation expresses the fact that $e^{-i \beta} W=\omega$ is real everywhere in the domain including its boundary. By differentiation of $\operatorname{Im}\left(e^{-i \beta} W\right)=0$ with respect to the outward normal $n$ one finds

$$
e^{-i \beta} W_{n}^{\prime}-e^{i \beta} \overline{W_{n}^{\prime}}=i \beta_{n}^{\prime}\left(e^{-i \beta} W+e^{i \beta} \bar{W}\right), \quad \zeta \in \Gamma .
$$

Taking (25) into account one can rewrite the right-hand side of (26) and present the second boundary condition in the form 


$$
e^{-i \beta} W_{n}^{\prime}-e^{i \beta} \overline{W_{n}^{\prime}}=2 i \beta_{n}^{\prime}(\zeta) e^{-i \beta} W, \quad \zeta \in \Gamma .
$$

The derivatives of the complex-valued function $f=f(\zeta)$ on the contour $\Gamma$ are defined as their limiting values. Respectively, the tangent and normal derivatives are presented as follows:

$$
\frac{\partial f}{\partial \boldsymbol{l}}=\zeta^{\prime} \lim _{z \rightarrow \zeta} \frac{\partial f}{\partial z}+\overline{\zeta^{\prime}} \lim _{z \rightarrow \zeta} \frac{\partial f}{\partial \bar{z}}, \quad \frac{\partial f}{\partial \boldsymbol{n}}=-i \zeta^{\prime} \lim _{z \rightarrow \zeta} \frac{\partial f}{\partial z}+i \overline{\bar{\zeta}^{\prime}} \lim _{z \rightarrow \zeta} \frac{\partial f}{\partial \bar{z}} .
$$

Here one should notice that the derivatives in the left-hand side are taken with respect to the arc length $s$, i.e. they can be expressed through the derivatives with respect to the complex variable $\zeta$ on the contour by the following relationship:

$$
\frac{\partial f}{\partial \boldsymbol{l}}=\frac{\partial f}{\partial s}=\frac{\partial f}{\partial \zeta^{\prime}}=e^{i \vartheta} \frac{\partial f}{\partial \zeta}, \quad \zeta \in \Gamma .
$$

By taking into account that $\zeta^{\prime}=\exp (i \vartheta)$ one can present the tangential and normal derivatives of the vector $W$ on the contour $\Gamma$ in the form

$$
\begin{gathered}
W_{l}^{\prime}=e^{i \vartheta}(\kappa \Phi(\zeta)-\overline{\Phi(\zeta)})-e^{-i \vartheta}\left(\overline{\zeta \Phi^{\prime}(\zeta)}+\overline{\Psi(\zeta)}\right), \quad \zeta \in \Gamma, \\
W_{n}^{\prime}=-i e^{i \vartheta}(\kappa \Phi(\zeta)-\overline{\Phi(\zeta)})-i e^{-i \vartheta}\left(\overline{\zeta \Phi^{\prime}(\zeta)}+\overline{\Psi(\zeta)}\right), \quad \zeta \in \Gamma,
\end{gathered}
$$

where $\Psi(\mathrm{z})$ is defined by (3) where $h^{\prime}=-e^{-2 i \vartheta} \bar{g}^{\prime}$. It is evident from (28) that

$$
W_{l}^{\prime}+i W_{n}^{\prime}=2 e^{i \vartheta}(\kappa \Phi(\zeta)-\overline{\Phi(\zeta)}), \quad W_{l}^{\prime}-i W_{n}^{\prime}=-2 e^{-i \vartheta}\left(\overline{\zeta \Phi^{\prime}(\zeta)}+\overline{\Psi(\zeta)}\right), \quad \zeta \in \Gamma
$$

The latter formulas can be rewritten by using complex conjugation of the second expression in (32) and by passing to the complex derivative using (29) that results in the following expressions:

$$
W_{z}^{\prime}=\kappa \Phi(\zeta)-\overline{\Phi(\zeta)}, \overline{W_{z}^{\prime}}=-\bar{\zeta} \Phi^{\prime}(\zeta)-\Psi(\zeta) .
$$

Note that these can be directly obtained by direct differentiation of (1). Formulas (32)-(33) provide the link between the tangential and normal derivatives and the boundary values of the derivatives with respect to $z$ and its conjugate on the contour $\Gamma$. It is evident that if the boundary value of any complex-valued function is known than the tangential derivative is also known and if its normal derivative is known then one can find the boundary values of the derivatives with respect to $z$.

Let us combine two real conditions (25) and (27) into one complex by differentiation of $\operatorname{Im}\left(e^{-i \beta} W\right)=\operatorname{Im}(\omega)=0$ with respect to the variable $z$. Similarly to (27) one finds

$$
e^{-i \beta} W_{z}^{\prime}-e^{i \beta} \overline{W_{z}^{\prime}}=2 i \beta_{z}^{\prime} e^{-i \beta} W, \quad \zeta \in \Gamma .
$$

However in contrast to (27) this form is now complex and combines two real conditions equivalent to (25) and (27), therefore it can be used for obtaining a complex SIE. 


\subsubsection{Complex singular integral equation}

Let us substitute the expression for $W$ into the right-hand side of (34) and (33) into the lefthand side of (34) to obtain the following boundary value problem for the determination of the holomorphic functions $\varphi, \psi$ and their derivatives

$$
e^{-i \beta}(\kappa \Phi(\zeta)-\overline{\Phi(\zeta)})+e^{i \beta}\left(\bar{\zeta} \Phi^{\prime}(\zeta)+\Psi(\zeta)\right)=2 i \beta_{z}^{\prime} e^{-i \beta}(\kappa \phi(\zeta)-z \overline{\Phi(\zeta)}-\overline{\psi(\zeta)}), \quad \zeta \in \Gamma .
$$

Now it is possible to derive a SIE by substituting the boundary values of the complex potentials into (35) followed by the application of the Sokhotski-Plemelj formulas. However, this would lead to a SIE that contains both unknown function $g(\zeta)$ and its derivative. No direct techniques for investigation of such SIEs are known, which necessitates further transformations in order to obtain a SIE of the Gakhov's-type mentioned above.

Selection of $h=\kappa \bar{g}$ followed by substitution of (8) and (9) into (25) which results in

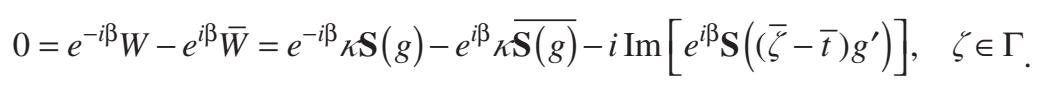

Note that the last term on the right-hand side is not singular.

By differentiation of (36) with respect to $\zeta$ one finds

$$
\begin{gathered}
2 i \beta_{\zeta}^{\prime}\left(e^{-i \beta} k \mathbf{S}(g)+e^{i \beta} \bar{k} \overline{\mathbf{S}(g)}\right)=e^{-i \beta}, \mathbf{S}\left(g^{\prime}\right)-e^{i \beta} \bar{\kappa} \overline{\mathbf{S}\left(e^{-2 i \vartheta} g^{\prime}\right)} \\
-i \frac{d}{d \zeta} \operatorname{Im}\left[e^{i \beta} \mathbf{S}\left((\bar{\zeta}-\bar{t}) g^{\prime}\right)\right], \quad \zeta \in \Gamma .
\end{gathered}
$$

This provides the relationship between the unknown function $g$ and its contour derivative $g$ ' that is further considered as the only unknown function after transformation of the righthand side of (34) and (35). The left-hand side of (35) is also transformed in view of the Sokhotski-Plemelj formulas, which eventually leads to the following complex SIE:

$$
\begin{gathered}
\left.e^{-i \beta}\left[ \pm\left(\kappa g^{\prime}-\bar{g}^{\prime}\right)+\mathbf{S}\left(g^{\prime}\right)-\overline{\mathbf{S}\left(g^{\prime}\right)}\right]+e^{i \beta}\left[ \pm e^{2 i \vartheta}\left(\kappa g^{\prime}-\bar{g}^{\prime}\right)+\overline{\mathbf{S}\left(e^{-2 i \vartheta}\left(\kappa g^{\prime}-\bar{g}^{\prime}\right)\right)}+\frac{d}{d \zeta} \mathbf{S}(\bar{\zeta}-\bar{t}) g^{\prime}\right)\right] \\
\left.-\kappa \frac{\beta_{z}^{\prime}}{\beta_{\zeta}^{\prime}}\left(e^{-i \beta} \mathbf{S}\left(g^{\prime}\right)-e^{i \beta} \overline{\mathbf{S}\left(e^{-2 i \vartheta} g^{\prime}\right)}-\frac{i}{\kappa} \frac{d}{d \zeta} \operatorname{Im}\left[e^{i \beta} \mathbf{S}(\bar{\zeta}-\bar{t}) g^{\prime}\right)\right]\right)=0, \quad \zeta \in \Gamma .
\end{gathered}
$$

Equation (38) represents the full complex SIE for an arbitrary counter for BVP2. It should be complemented by the integral conditions expressing the fact that the function $g$ returns to its initial value after the complete traverse of the contour,

$$
\int_{\Gamma} g^{\prime}(t) d t=0 .
$$

\subsubsection{The SIE for half-plane}

It has been mentioned above that solvability of the BVP can be investigated for the case of half-plane, for which the formulas of the previous section can be readily simplified by assuming $\vartheta=0, \operatorname{Im} \zeta=0$, in particular the singular operator assumes the form as shown in (18). It obeys the properties shown in (5) and (19). The former is valid for any closed contour, while the latter is valid only for the real axis. It is also evident that due to (39) one can conclude that 


$$
\mathbf{S}\left((\bar{\zeta}-\bar{t}) g^{\prime}\right)=\mathbf{S}\left((x-t) g^{\prime}\right)=0
$$

We further consider the case of upper half-plane and therefore the SIE assumes the following form :

$$
\begin{gathered}
\left(e^{-i \beta}+e^{i \beta}\right)\left(\kappa g^{\prime}-\bar{g}^{\prime}\right)+\kappa\left(e^{-i \beta}-e^{i \beta}-\frac{\beta_{z}^{\prime}}{\beta_{x}^{\prime}} e^{-i \beta}\right) \mathbf{S}\left(g^{\prime}\right)-\left(e^{-i \beta}+e^{i \beta}-\kappa \frac{\beta_{z}^{\prime}}{\beta_{\mathrm{x}}^{\prime}} e^{i \beta}\right) \\
\overline{\mathbf{S}\left(g^{\prime}\right)}=0,|x|<\infty .
\end{gathered}
$$

This equation is the homogeneous dominant SIE, a part of the full SIE of the form investigated by Gakhov [9]. Namely

$$
a g+b \bar{g}+c \mathbf{S} g+d \overline{\mathbf{S} g}+\mathbf{K} g+\overline{\mathbf{L} g}=f .
$$

Here $f=0$ and the coefficients $a, b, c$ and $d$ are known continuous functions of the complex contour variable specified as follows:

$$
\begin{gathered}
a=\kappa\left(e^{-i \beta}+e^{i \beta}\right), b=-e^{-i \beta}-e^{i \beta}, c=\kappa\left(e^{-i \beta}-e^{i \beta}-\frac{\beta_{z}^{\prime}}{\beta_{x}^{\prime}} e^{-i \beta}\right), \\
d=-e^{-i \beta}-e^{i \beta}+\kappa \frac{\beta_{z}^{\prime}}{\beta_{x}^{\prime}} e^{i \beta} .
\end{gathered}
$$

The regular operators $\mathbf{K g}$ and $\mathbf{L g}$ can be found from the full SIE (38), but in the case of half-plane they vanish.

It has been shown [9] that the solvability of SIE (42) is determined by the index calculated through the increment of the argument of the complex valued function $G$ after the complete traverse of $\Gamma$ in the counter-clockwise direction as follows:

$$
\begin{aligned}
& 2 \mathrm{~K}_{2} \equiv \operatorname{Index}(G)=\left.\frac{1}{2 \pi i}[\ln (G)]\right|_{\Gamma}=\left.\frac{1}{2 \pi}[\arg (G)]\right|_{\Gamma} \\
& G=\frac{A-i B}{A+i B}, \quad A-i B=(\bar{a}+\bar{c})(a-c)+(b+d)(\bar{d}-\bar{b})
\end{aligned}
$$

If $2 \mathrm{~K}_{2}>0$ (the coefficient 2 is introduced for convenience), then the solution of the homogeneous equation SIE $(f=0)$ depends on $2 \mathrm{~K}_{2}$ complex constants or $4 \mathrm{~K}_{2}$ real constants, otherwise the SIE does not have solutions, see [9].

\subsubsection{Special case of boundary conditions}

Let us consider the case $\beta_{n}^{\prime}(\zeta)=0$, which can be important for some applications in geomechanics. In this case the coefficients $a, b, c$ and $d$ become

$$
a=\kappa\left(e^{-i \beta}+e^{i \beta}\right), b=-e^{-i \beta}-e^{i \beta}, c=-\kappa e^{i \beta}, d=(\kappa-1) e^{i \beta}-e^{-i \beta}
$$

Therefore,

$$
A-i B=4 \kappa \cos \beta\left(\kappa e^{i \beta}-e^{-i \beta}\right)
$$


and consequently

$$
G=\frac{A-i B}{A+i B}=e^{2 i \beta} \frac{\kappa e^{i \beta}-e^{-2 i \beta}}{\kappa-e^{2 i \beta}} .
$$

Since $\kappa>1$ the fraction in (47) does not affect the index as its real part is always positive, therefore, the index is found as follows:

$$
2 \mathrm{~K}_{2} \equiv \operatorname{Index}(G)=\frac{1}{2 \pi i}\left[\ln (\exp (2 i \beta)]_{\Gamma}=\left[\frac{\beta}{\pi}\right]_{\Gamma} .\right.
$$

This result indicates that the index depends on the displacement orientations only, i.e. on the angle $\beta$, whose increment (after the complete traverse of $\Gamma$ in the positive direction) should be positive in order to provide a solution of the considered BVP. If $2 K_{2} \leq 0$, no bounded (non-trivial) solutions exist. For $2 \mathrm{~K}_{2}>0$ the solution of (43) for the special case of the coefficients (47) includes an arbitrary polynomial of $2 \mathrm{~K}_{2}-1$ degree, which means that the solution is controlled by up to $4 \mathrm{~K}_{2}$ real parameters.

It is worth noting that the results regarding the solvability for both problems BVP1 and BVP2 are quite similar despite essential differences in the governing equations they satisfy.

\subsection{Problem $(\alpha, \beta)$ for half-plane}

Consider the upper half-plane. Then the indices \pm can be omitted and one can write the BC (13) and (14) on the real axis in the form

$$
\begin{aligned}
& \operatorname{Im}\left(e^{-i \alpha} D\right)=0 \quad \Leftrightarrow \quad e^{-i \alpha} D=e^{i \alpha} \bar{D}, \quad \text { on } \quad \Gamma=(x:-\infty<x<\infty), \\
& \operatorname{Im}\left(e^{-i \beta} W\right)=0 \quad \Leftrightarrow \quad e^{-i \beta} W=e^{i \alpha} \bar{W}, \quad \text { on } \quad \Gamma=(x:-\infty<x<\infty) .
\end{aligned}
$$

We will seek solutions that provide continuity of the stress vector across the real axis, which follows from (20) and (21) when $h=-\bar{g}$, which leads to the following boundary values on the real axis:

$$
\begin{aligned}
& D^{+}=-\mu-\mathbf{S}(\mu), \quad 2 \mu=g^{\prime}+\bar{g}^{\prime}, \\
& 2 G W^{+}=(\kappa+1) g+(\kappa-1) \mathbf{S}(g) .
\end{aligned}
$$

Substitution of (51) into (49) and (52) into (50) with the use of (19) leads to the system of SIEs in the form

$$
\begin{aligned}
& e^{-i \alpha}[\mu+\mathbf{S}(\mu)]-e^{i \alpha}[\mu-\mathbf{S}(\mu)]=0, \\
& e^{-i \beta}[(\kappa+1) g+(\kappa-1) \mathbf{S}(g)]-e^{i \beta}[(\kappa+1) \bar{g}-(\kappa-1) \mathbf{S}(\bar{g})]=0 .
\end{aligned}
$$

By introducing the following notations:

$$
g=\mathrm{M}+i \Lambda, \quad \mathrm{M}^{\prime}=\mu, \quad \operatorname{Im}(\mathrm{M})=\operatorname{Im}(\Lambda)=\operatorname{Im}(\mu)=0,
$$

one can present (53) as follows: 


$$
\begin{aligned}
& \sin (\alpha) \mathbf{M}^{\prime}+i \cos (\alpha) \mathbf{S}\left(\mathbf{M}^{\prime}\right)=0 \\
& (\kappa+1) \cos (\beta) \Lambda-i(\kappa-1) \sin (\beta) \mathbf{S}(\Lambda)=f .
\end{aligned}
$$

Here the right-hand side of the latter equation depends on the solution of the former equation. It has the form

$$
f=(\kappa+1) \sin (\beta) \mathbf{M}+i(\kappa-1) \cos (\beta) \mathbf{S}(\mathbf{M}) .
$$

The system of SIE (55) can be reduced to two Riemann BVPs on the real axis. The former SIE in (55) is homogeneous; it is reduced to the homogeneous Riemann BVP

$$
A^{+}=-e^{2 i \alpha} A^{-} \text {. }
$$

The latter equation in (55) is equivalent to the following non-homogeneous Riemann BVP

$$
B^{+}=e^{2 i(\beta-\varsigma)} B^{-}+\frac{e^{i \beta}}{\kappa+e^{2 i \beta}} f, \quad \varsigma=\arg \left(\kappa+e^{2 i \beta}\right) .
$$

The indexes of these BVPs are determined as follows:

$$
\begin{gathered}
2 \mathrm{~K}=\operatorname{Index}\left(e^{2 i \alpha}\right)=\operatorname{Index}\left(e^{-4 i \theta}\right)=-\left.\frac{2}{\pi}[\theta]\right|_{-\infty} ^{\infty}, \\
2 \mathrm{~N}=\operatorname{Index}\left(e^{2 i \beta}\right)=\left.\frac{1}{\pi}[\beta]\right|_{-\infty} ^{\infty}
\end{gathered}
$$

In (60) it has been taken into account that $\operatorname{Index}\left(e^{i \varsigma}\right)=0$ as $\operatorname{Re}\left(\kappa+e^{2 i \beta}\right)>0$.

Further on we employ the general solution from Gakhov [9] to seek solutions of (57)-(58) vanishing at infinity. These solutions obviously depend on the indexes (59)-(60), respectively. The solution for the derivative of the function $\mathrm{M}$ is found in the form:

$$
\mathrm{M}^{\prime}(x)=2 \cos \alpha(x) \frac{P_{2 \mathrm{~K}-1}(x)}{\left(x^{2}+1\right)^{\mathrm{K}}} e^{-\mathrm{S}\left[\mathrm{K} \ln \left(\frac{t-i}{t+i}\right)+i \alpha(t)\right]} .
$$

Here the exponential function is real-valued, thus the coefficients of the polynomial $P_{2 \mathrm{~K}-1}$ should be real as well to provide real-valuedness of the sought function. Therefore, for positively defined index (59) the solution depends on $2 \mathrm{~K}$ arbitrary real constants.

Let us solve the second SIE in (55) assuming provisionally that $2 \mathrm{~N}>0$ and the function $f$ specified by (56) is known. This solution (vanishing at infinity) is found from the general solution provided in [9] as follows:

$$
\Lambda(x)=\cos (\beta(x)-\varsigma(x)) f(x)+i \sin (\beta(x)-\varsigma(x))\left[Z(x) \mathbf{S}\left(\frac{f(t)}{Z(t)}\right)-\frac{\tilde{P}_{2 \mathrm{~N}-1}(x)}{\left(x^{2}+1\right)^{\mathrm{N}}}\right] .
$$

Here for briefness the following notation was introduced

$$
Z(x)=\exp \left[-\mathbf{S}\left(\mathrm{N} \ln \frac{t-i}{t+i}-i \beta+i \varsigma\right)\right] .
$$

It should be noted that the function $Z(\mathrm{x})$ is real-valued because the singular integral is applied to the purely imaginary term in the parentheses and it hence is real-valued. Then it becomes evident that the first term in the square brackets of (62) is purely imaginary, 
therefore, the second term should be purely imaginary as well to provide real-valuedness of the sought function $\Lambda$. Hence all the coefficients of the polynomial $\tilde{P}_{2 \mathrm{~N}-1}(x)$ are purely imaginary.

The total index of the Riemann problems (57) and (58) is the sum of partial indexes

$$
2 \mathrm{~K}_{3}=2 \mathrm{~K}+2 \mathrm{~N}=\operatorname{Index}\left(e^{2 i \alpha}\right)+\operatorname{Index}\left(e^{2 i \beta}\right)=\left.\frac{1}{\pi}[\beta+\alpha]\right|_{-\infty} ^{\infty}=\left.\frac{1}{\pi}[\beta-2 \theta]\right|_{-\infty} ^{\infty} .
$$

However, the general number of arbitrary real constants entering the solution of SIE is $2 \mathrm{~K}+2 \mathrm{~N}+1$, where an extra constant generated by integration of (61) should be added in order to find the right-hand side of the non-homogeneous equation in the system. This number is the maximum possible number of arbitrary constant provided that both indexes are positive. If one of them is negative, say $2 \mathrm{~N}<0$, then solutions exist if $(-2 \mathrm{~N}-1)$ integral conditions are imposed on the right-hand side of the non-homogeneous equation of the system (see detail in Gakhov [9]). They can be always satisfied provided that $2 \mathrm{~K}>-2 \mathrm{~N}-1$. Therefore, for solvability of the system of SIE (55) it is necessary and sufficient that the cumulative index $2 \mathrm{~K}+2 \mathrm{~N}+1$ is positive. Even more the number of solutions is odd because both indices determined by formulas (59) and (60) are even numbers, which is evident from the fact that the stress and displacement orientations attaint their initial values after the complete traverse of the contour.

It should noted that the number of arbitrary constants affecting the stresses can be reduced to $2 \mathrm{~K}+2 \mathrm{~N}$, bearing in mind that three constants can be eliminated as translation and rotation of the rigid body do not affect stresses. As soon as the displacements at infinity were zero one needs to satisfy the only additional condition on rotation, which would fix one of the coefficient of the polynomial $\tilde{P}_{2 \mathrm{~N}-1}(x)$ from the condition $\operatorname{Im}(\Phi(0))=0$.

\section{NUMERICAL APPROACH}

The problems considered above have been reduced to a system of two real equations, see (23) and (45), or to a single complex equation (38) that belongs to the type of SIE shown in (42) investigated by Gakhov [9]. In all cases at least one of the equations is homogeneous. This fact restricts the direct application of any boundary element technique to the homogeneous dominant SIE as it would produce only a trivial solution due to $f=0$. Therefore it is necessary to transform this SIE such that its right-hand side becomes non-homogeneous and none of the homogeneous solutions is lost. Moreover, the standard BEM techniques cannot be applied directly to a non-homogeneous SIE with non-zero index as this would result in incomplete solution due to neglecting of the homogeneous part of the total solution. Therefore, the numerical approaches for eqns (23), (38) and (45) should address the fact that these SIE can have multiple solutions the number of which is determined in accordance with the index of the corresponding Riemann BVP. Below we outline a possible numerical approach that can be applied as to the case of half-plane (that lead to the consideration of the homogeneous dominant SIE) as well as for arbitrary contours (full SIE, e.g. (38)).

It has already been shown in [9] that application of the following integral operator

$$
\boldsymbol{\Theta}(.)=\bar{a} \mathbf{I}(.)-b \overline{\mathbf{I}(.)}-\bar{c} \mathbf{S}(.)-d \overline{\mathbf{S}(.)}
$$

(I is the identical operator, i.e. $\mathbf{I} f=f$ ) reduces the dominant equation (42) (as well as the full SIE) to the form

$$
\begin{gathered}
A g^{\prime}-i B \mathbf{S} g^{\prime}+\mathbf{R} \operatorname{eg}\left(g^{\prime}, \bar{g}^{\prime}\right)=F \\
A=a \bar{a}-b \bar{b}-c \bar{c}+d \bar{d}, \quad i B=\bar{a} c-a \bar{c}+\bar{b} d-b \bar{d}, \quad \operatorname{Im}(A)=\operatorname{Im}(B)=0 .
\end{gathered}
$$


where $F=\boldsymbol{\Theta}(f)$ is known. The regular integral $\operatorname{Reg}($.$) reflects all regular terms as those$ obtained by the change of the order of integration after application of (65) to the dominant part of (42) as well as regular integrals $\mathbf{K}$ and $\mathbf{L}$ in (42) or any other regular terms that are included in the full system of equations for arbitrary domains consisting of two real SIEs.

We further seek the solution of (66) as the sum of two functions

$$
g^{\prime}=g_{0}^{\prime}+g_{1}^{\prime}
$$

Here $g_{0}^{\prime}$ is the general solution of the homogeneous dominant equation

$$
A g_{0}-i B \mathbf{S} g_{0}=0,
$$

and $g_{1}^{\prime}$ is any particular solution of the non-homogeneous SIE

$$
A g_{1}^{\prime}-i B \mathbf{S} g_{1}^{\prime}+\operatorname{Reg}\left(g_{1}^{\prime}, \bar{g}_{1}^{\prime}\right)=F-\operatorname{Reg}\left(g_{0}^{\prime}, \bar{g}_{0}^{\prime}\right) .
$$

where the right-hand side contains the analytical solution of SIE (68); it includes all linearly independent solutions of (68) and hence (69) is not homogeneous anymore even if $F=0$. Therefore, any proper boundary element method can be applied to find the numerical solution of the non-homogeneous SIE (69). Finally, the sum in (67) determines the solution of the homogeneous dominant SIE, where the analytical part $g_{0}^{\prime}$ is found as described in Section 4. Besides, when dealing with BVP2, the solution for the contour derivative of the displacement vector is found from the following relationship for the displacement vector

$$
W(\zeta)=\kappa \mathbf{S}(g)-\frac{1}{2} \overline{\mathbf{S}\left((\bar{\zeta}-\bar{t}) g^{\prime}\right)} .
$$

In the case of the system of two real SIE the approach above remains but should be applied to both SIEs in (23) and (45) followed by a procedure for the selection of the coefficients of the polynomials to provide real valuedness of the solution. It should be noted that this approach without any modifications can be applied for solving the system of two full SIEs as well.

\section{CLOSURE}

The incorrectly posed BVPs considered in this study are useful not only for geomechanics, where they originated from, but also for solid mechanics, computational mechanics and geophysics. This is especially important in the cases when the standard BVPs fail due to uncertainties in boundary conditions or in the cases where the influence of gauges measuring displacements/strains on their real values cannot be filtered out. One can find quite a few applications of the proposed approach provided that they can be considered in the framework of the plane elastic problem which is the main limitation of the method.

It is evident that no changes in the principal stress orientations are observed if a constant mean stress is added to a solution of a plane problem or if all stress components are multiplied by a positive numbers. This indicates non-uniqueness of non-classical BVP1 and BVP3. However, these simplest modifications of the stress fields are not the only possible ones as has been shown in the present study where we revealed what parameter (Index) controls the number of independent solutions.

In this paper, we presented a general approach for investigation of the solvability of incorrectly posed BVPs of plane elasticity formulated in terms of stress and displacement orientations. The method is based on the reduction of all considered problems, BVP1-BVP3, to a corresponding Riemann BVPs. This allows one to determine the number of linearly 
independent particular solutions of by calculating the index of the Riemann problem. Therefore, all the BVP considered may have non-unique solutions or be unsolvable if the index ix negative.

The method proposed by Gakhov [9] for the investigation of the complex SIE (42) has been generalised for more complex cases by reducing the SIE containing both the unknown function and its derivatives to the form of SIE in (42) by manipulations with the contour derivatives (Subsections 4.1.2 and 4.1.3) and by introduction of auxiliary unknown functions (Subsection 4.2). This has allowed us to fully investigate the solvability of BVP2 and BVP3 on the basis of Gakhov's method.

It is worth noting that despite apparent similarity in formulations of BVP1 and BVP2, these problems are essentially different as they deal with the functions that satisfy different governing equations. Indeed the function $D$ is bi-holomorphic while $W$ satisfy the Lame equation if complex form (22). The corresponding system of SIE are also essentially different for these problems. However the results regarding solvability look quite similar in both cases the number of partial solutions is defined by the number of rotations either the principal directions of the stress tensor (as in BVP1) or the displacement vector (as in BVP2). Besides the solvability of these BVP does not depend on the behaviour of the normal derivative $\alpha_{\mathbf{n}}^{\prime}$, $\beta_{n}^{\prime}$. The solvability of BVP3 is defined by the cumulative index of the problem BVP1 and BVP2, which indicates that BVP3 can posses multiple solutions even if one of the problems (BVP1 or BVP2) do not have (bounded) solutions. Analysis of such situations is left for future work.

A numerical approach capable of dealing with both homogeneous and non-homogeneous SIEs has been proposed. It can be applied to either of formulations as those leading to real systems of two SIEs or to a single complex SIE.

\section{ACKNOWLEDGEMENTS}

The author is thankful to the Russian Foundation for Basic Research that supported this work by the Grant 16-01-00260.

\section{REFERENCES}

[1] Galybin, A.N., Cauchy BVP for elastic half-plane posed in displacement orientations. WIT Transactions on Engineering Sciences, 122, WIT Press, pp. 201-208, 2018.

[2] Galybin, A.N., Boundary value problems for elastic half-planes posed in terms of stress and displacement orientations. International Journal of Computational Methods and Experimental Measurements, 6(6), pp. 1173-1181, 2018. https://doi.org/10.2495/ cmem-v6-n6-1173-1181

[3] Galybin, A.N. \& Mukhamediev, S.A., Plane elastic boundary value problem posed on orientation of principal stresses. Journal of the Mechanics and Physics of Solids. 47(11), pp. 2381-2409, 1999. https://doi.org/10.1016/s0022-5096(99)00032-0

[4] Galybin, A.N. \& Mukhamediev, S.A., Integral equations for elastic problems posed in principal directions: application for adjacent domains. WIT Transactions on Modelling and Simulation, 42, WIT Press, Southampton, UK, pp. 51-60, 2006.

[5] Galybin, A.N., Boundary value problems posed in terms of stress orientations for plane crack systems. Proceedings of 12th International Conference on Fracture. Ottawa, on CD-ROM, paper T02.007 (fin 00560), p. 7, 2009.

[6] Galybin, A.N., Boundary value problems of plane elasticity involving orientations of displacements and tractions. Journal of Elasticity, 102(1), pp. 15-30, 2011. https://doi. org/10.1007/s10659-010-9259-4 
[7] Galybin, A.N., Boundary integral equations for plane elastic problems posed in terms of stress orientations. Proc. of the 8th UK Conference on Boundary Integral Methods, ed. D. Lesnic, University of Leeds, UK, , pp. 194-198, 2011.

[8] Galybin, A.N., On solvability of the BVP formulated in terms of displacement orientations on the interface between dissimilar elastic materials. International Journal of Computational Methods and Experimental Measurements, 5(3), pp. 369-376, 2017. https://doi.org/10.2495/cmem-v5-n3-369-376

[9] Gakhov, F.D., Boundary Value Problems, 3rd Russian edn, Moscow, Nauka (see also the translation of the first edition by Pergamon Press, 1966.) 1977.

[10] Muskhelishvili, N.I., Some Basic Problems of the Mathematical Theory of Elasticity, ed. P. Noordhoff, Groningen, the Netherlands, 1963.

[11] Linkov, A.M., Boundary Integral Equations in Elasticity Theory, Springer Netherlands, 2002. 